\title{
El cambio climático como problema global: herramientas jurídicas para conciliar ambición y eficacia y el rol del Acuerdo de París
}

Francisca Aguayo Armijo*

Para citar este artículo: Aguayo Armijo, F. (2022). El cambio climático como problema global: herramientas jurídicas para conciliar ambición y eficacia y el rol del Acuerdo de París. Desafíos, 34(1), 1-16. https://doi.org/10.12804/revistas.urosario.edu.co/desafios/a.10831

\section{Introducción}

Uno de los principales desafíos en la lucha contra el cambio climático ha sido lograr un consenso internacional universal en torno a un régimen jurídico de reducción de emisiones de gases de efecto invernadero aplicable a todos los países. Ante la diversidad de intereses políticos en juego, reflejados en la pluralidad de grupos negociadores en la materia, la cuestión de cómo reflejar en los marcos normativos compromisos aceptables de manera universal ha constituido durante casi tres décadas uno de los principales obstáculos para alcanzar una eficacia real de la acción internacional ante un problema por naturaleza global, transfronterizo y multidimensional.

Cabe recordar que el régimen jurídico específico en materia de lucha contra el cambio climático se inició con la adopción de la Convención Marco de las Naciones Unidas sobre el Cambio Climático (CMNUCC) en la Conferencia de las Naciones Unidas sobre el Medio Ambiente

\footnotetext{
* Social Science Research Council. Correo electrónico: francisca.aguayo.a@gmail.com. ORCID: https://orcid.org/0000-0001-7787-226X
} 
y Desarrollo (conocida como Cumbre para la Tierra), celebrada en Río de Janeiro en 1992. En esta instancia, los Estados miembros adoptaron también la Declaración de Río sobre el Medio Ambiente y Desarrollo, en la que se profundizó en el compromiso internacional acordado en la Conferencia de las Naciones Unidas sobre el Medio Humano celebrada en Estocolmo en 1972, que, como primera conferencia enfocada en problemas ambientales, posicionó el tema en la agenda internacional. En los años posteriores a la Conferencia de Estocolmo se adoptaron instrumentos cruciales en materia ambiental, vinculados directamente con la lucha contra el cambio climático, dentro de los que destacan la Convención de Viena para la Protección de la Capa de Ozono de 1985 y el Protocolo de Montreal relativo a las sustancias que agotan la capa de ozono de 1987, ratificados de manera universal en la antesala de la adopción de la Declaración de Río y la CMNUCC.

Al igual que la Convención de Viena, la CMNUCC es una convención marco que establece, en un instrumento jurídicamente vinculante, objetivos, principios y obligaciones generales destinados a su operacionalización y precisión progresiva mediante la adopción de acuerdos posteriores. Así, las negociaciones desplegadas durante las últimas décadas en el marco de la CMNUCC se han enfocado en la adopción de instrumentos diversos que, basándose en una diversidad de herramientas jurídicas, han intentado precisar el régimen de reducción de emisiones de gases de efecto invernadero. El desafío, aún presente en la actualidad, ha sido conciliar la ambición de la respuesta internacional ante la urgencia del problema del cambio climático con la necesidad de garantizar una aceptación universal del régimen que lo dote de eficacia real.

En este contexto, el presente trabajo analiza la diversidad de herramientas ofrecidas por el derecho internacional que han sido utilizadas a lo largo de las negociaciones en el marco de la CMNUCC para poner de relieve las soluciones híbridas que incorpora el Acuerdo de París, incluida la interacción entre el derecho "duro" y el soft law (o derecho "blando"). Se plantea que, en base a las lecciones generadas a partir de las experiencias previas, el Acuerdo de París aporta una flexibilidad 
que podría lograr una conciliación real y efectiva entre la ambición de la respuesta internacional y la necesidad de garantizar una aceptación universal del régimen jurídico, que se constituye como el resultado de equilibrios delicados y factibles entre intereses políticos diversos.

\section{La búsqueda de una conciliación mediante herramientas jurídicas diversas}

Múltiples razones, tanto políticas como técnicas, explican la utilización de diversas herramientas jurídicas en las negociaciones intergubernamentales en materia ambiental y de lucha contra el cambio climático. Por una parte, y de manera fundamental, destaca la profunda diversidad y divergencia de intereses políticos, que no puede reducirse a diferencias entre países desarrollados y en desarrollo, lo que se ve reflejado en la diversidad de grupos negociadores que han sido creados en el marco de la CMNUCC (Carbon Brief, s. f.; Rajamani, 2020). Se han constituido grupos negociadores tanto dentro del Grupo de los 77 y China — marcando las diferencias de intereses entre países en desarrollo_ como entre países desarrollados, así como coaliciones transversales entre países desarrollados y en desarrollo para impulsar una acción progresista. Por otra parte, en lo técnico, se han utilizado diversas herramientas jurídicas para introducir un grado de flexibilidad en los marcos normativos que permita adaptarlos progresivamente a la evolución del conocimiento científico.

\section{Las técnicas de negociación}

En lo procedimental, las negociaciones intergubernamentales en el marco de la CMNUCC se han basado en la técnica de consenso (Boyle \& Ghaleigh, 2016). El consenso, utilizado de manera amplia en distintos ámbitos de la acción internacional, consiste en lograr un acuerdo común aceptable por todos los Estados, manifestado por la ausencia de oposición al texto y, por consiguiente, de votación. El consenso permite así reflejar, en cierta medida, las distintas perspectivas de los países, considerando a la vez la mayor influencia que tienen las grandes potencias durante las negociaciones. En este sentido, estrechamente vinculada a la posibilidad 
de lograr un instrumento de consenso, la técnica del package deal (similar a un paquete todo incluido) asegura que el instrumento sea concebido, interpretado y aplicado como un acuerdo de conjunto, cuyas normas están vinculadas entre sí logrando reflejar equilibrios políticos delicados. Se entiende que sin esta visión de conjunto en que las normas se equilibran las unas con las otras, el texto no hubiese podido ser adoptado.

Si bien la utilización de estas técnicas han permitido lograr acuerdos en un ámbito caracterizado por intereses políticos diversos, el uso del consenso plantea la interrogación sobre la dilución del lenguaje en los instrumentos adoptados, en tanto se trata de un acuerdo que debe ser aceptable por una diversidad de Estados que constituye, por lo tanto, un mínimo común denominador. Sin embargo, es aquí donde se debe considerar la necesidad de conciliar el avance jurídico logrado por un instrumento con su eficacia real, que, ante un desafío por naturaleza global como lo es el cambio climático, solo puede lograrse mediante una participación universal en el régimen normativo. Más aún, tanto la técnica del consenso como del package deal fomentan en cierta medida el carácter inclusivo de las negociaciones, en tanto las visiones de todos los Estados deben ser consideradas para acordar un texto que requiere, simultáneamente, una flexibilidad en las negociaciones para reflejar esta diversidad de intereses, equilibrada por la lectura del texto en su conjunto.

\section{Los instrumentos adoptados en las negociaciones}

En lo relativo a los instrumentos adoptados, cabe destacar que en materia ambiental han proliferado marcos diversos producto de las negociaciones intergubernamentales y de la acción de organismos internacionales. A los numerosos tratados bilaterales y multilaterales adoptados por los Estados se suman una serie de instrumentos sin carácter jurídico vinculante, como declaraciones y resoluciones adoptadas en el marco de la Asamblea General de las Naciones Unidas, por citar solo algunos, e instrumentos adoptados por organismos internacionales. Este último fenómeno se ha visto reflejado en particular en la actividad del Programa de las Naciones Unidas para el Medio Ambiente (PNUMA), que ha participado en los procesos normativos 
intergubernamentales mediante la elaboración de instrumentos diversos como líneas directrices y guías de implementación.

Esta pluralidad de instrumentos constituye, en gran medida, un reflejo de la riqueza actual de las herramientas ofrecidas por el derecho internacional y de su evolución hacia una escala de normatividad relativa, que matiza la oposición entre instrumentos jurídicamente vinculantes e instrumentos de soft law o derecho "blando" (Pellet, 2018). Utilizado en la actualidad de manera transversal en prácticamente todos los ámbitos de la acción internacional, el soft law constituye una herramienta jurídica de particular importancia en el área ambiental.

Si bien los instrumentos de soft law negociados a nivel intergubernamental carecen de carácter obligatorio, están dotados de fines normativos e interactúan con los procesos de elaboración e implementación del derecho "duro" de manera estrecha, por lo que constituyen un complemento particularmente útil en materia ambiental. El soft law ha permitido en numerosas ocasiones lograr un acuerdo en un área en que diversos intereses políticos y económicos entran en juego y respecto de los cuales, en algunas instancias, ha sido más factible adoptar instrumentos en que los Estados no asumen obligaciones. Además, este otorga una flexibilidad mayor para modificar las normas - por ejemplo, mediante la adopción de une nueva resolución de la Asamblea General o la actualización de líneas directrices-, permitiendo una adaptación más rápida de los marcos normativos ambientales a la evolución científica, en contraste con los largos procesos de elaboración y ratificación de los tratados. Así, el soft law constituye un complemento importante de las normas de carácter jurídicamente vinculante, basado en interacciones múltiples y complementarias en que la elección del instrumento dependerá más bien de su finalidad y de la posibilidad de avanzar de una manera factible en la acción internacional.

Esta escala normativa se ha visto reflejada en el área climática en que, en el marco de las negociaciones para implementar la CMNUCC, se han adoptado acuerdos jurídicamente vinculantes, como el Protocolo de Kioto de 1997 y el Acuerdo de París de 2015, e instrumentos de soft law cuando no ha sido factible lograr un acuerdo vinculante, como 
el Acuerdo de Copenhague de 2009 y los Acuerdos de Cancún de 2010 (tabla 1).

Tabla 1. Principales instrumentos adoptados en el marco de la cmNucc

\begin{tabular}{|c|c|c|}
\hline Instrumento & $\begin{array}{c}\text { Fecha de adopción (y de entrada } \\
\text { en vigor si el instrumento es } \\
\text { vinculante) }\end{array}$ & $\begin{array}{l}\text { Valor jurídico del } \\
\text { instrumento }\end{array}$ \\
\hline $\begin{array}{l}\text { Convención Marco de las } \\
\text { Naciones Unidas sobre el } \\
\text { Cambio Climático }\end{array}$ & $\begin{array}{l}9 \text { de mayo } 1992 \text { (en vigencia el } 21 \\
\text { de marzo de 1994) }\end{array}$ & Vinculante \\
\hline Protocolo de Kioto & $\begin{array}{l}11 \text { de diciembre de } 1997 \text { (en } \\
\text { vigencia el } 16 \text { de febrero de 2005) }\end{array}$ & Vinculante \\
\hline Acuerdo de Copenhague & 18 de diciembre de 2009 & Soft law \\
\hline Acuerdos de Cancún & 11 de diciembre de 2010 & Soft law \\
\hline $\begin{array}{l}\text { Enmienda de Doha al } \\
\text { Protocolo de Kioto }\end{array}$ & $\begin{array}{l}8 \text { de diciembre de } 2012 \text { (en } \\
\text { vigencia el } 31 \text { de diciembre de } \\
2020 \text { ) }\end{array}$ & Vinculante \\
\hline Acuerdo de París & $\begin{array}{l}12 \text { de diciembre de } 2015 \text { (en } \\
\text { vigencia el } 4 \text { de noviembre de } \\
2016 \text { ) }\end{array}$ & Vinculante \\
\hline
\end{tabular}

Fuente: elaboración propia

Además de la fuerza jurídica del instrumento en sí, se ha experimentado, en particular en el caso del Acuerdo de París, con un contenido híbrido respecto de la obligatoriedad de las disposiciones en función de la posibilidad de lograr un acuerdo sobre normas obligatorias solo en algunos aspectos del régimen jurídico. Esto refleja el uso del soft law en el contenido del tratado como complemento de las obligaciones de carácter vinculante (Rajamani, 2016). Asimismo, respecto del contenido de estas obligaciones, el derecho internacional ofrece matices importantes al servicio del consenso, otorgando la posibilidad de adoptar obligaciones de medios o de resultado, así como obligaciones de carácter procedimental o sustantivo, variedad que ha sido ampliamente utilizada en el régimen internacional de lucha contra el cambio climático.

En esta misma línea, reflejando las posibilidades que ofrecen las herramientas jurídicas para lograr acuerdos mínimos ante intereses políticos diversos, se encuentra la utilización de las convenciones marco en 
materia ambiental, como la CMNUCC. La adopción de una convención marco permite establecer un acuerdo en torno a objetivos y principios comunes, así como obligaciones de carácter general y postergar la negociación de las normas de operacionalización, probablemente más difíciles e incluso imposibles de acordar al momento de adopción de la convención. De manera general, estas negociaciones posteriores tienen lugar en el marco de las Conferencias de las Partes (COP) a la convención - la más conocida es la COP en materia climática-, que permiten avanzar progresivamente en la implementación de los acuerdos logrados.

El ejemplo, por excelencia, del éxito que se puede alcanzar mediante este proceso normativo progresivo es logrado por la Convención de Viena sobre la Protección de la Capa de Ozono, en el marco de la cual se adoptó el Protocolo de Montreal relativo a las sustancias que agotan la capa de ozono. Ratificado de manera universal, el Protocolo de Montreal constituye un ejemplo emblemático de un instrumento internacional exitoso en el área ambiental, que contiene obligaciones internacionales en términos de porcentajes de reducción de emisiones destinadas a todos los países y en un calendario definido a nivel internacional — con mayor flexibilidad para los países en desarrollo_-, que ha logrado frenar el daño causado a la capa de ozono e incluso avanzar en una cierta recuperación. Con el transcurso del tiempo, debido tanto a la evolución científica como al reforzamiento de la acción internacional, por ejemplo, en materia de financiamiento para países en desarrollo, se ha continuado el avance progresivo del marco normativo mediante las enmiendas del Protocolo adoptadas en Londres (1990), Copenhague (1992), Montreal (1997), Beijing (1999) y Kigali (2016).

Cabe destacar, sin embargo, que la utilización de las convenciones marco conlleva a su vez un riesgo de fragmentación de los regímenes de implementación. En el plano jurídico, los protocolos y acuerdos posteriores constituyen un nuevo tratado internacional, en el que los Estados parte de la convención marco no tienen obligación alguna de participar. Por esto, cuando los instrumentos posteriores son ratificados de manera universal, como ha sucedido en el marco de la Convención de Viena, se puede lograr un avance significativo 
y real en la acción internacional. Este no ha sido históricamente el caso de la CMNUCC, que en su primer intento de operacionalización, mediante obligaciones jurídicas acordadas en el Protocolo de Kioto de 1997, se enfrentó a dificultades fundamentales de implementación y ratificación. Esta experiencia y las negociaciones posteriores para actualizar el marco normativo explican los equilibrios introducidos por el Acuerdo de París para lograr una conciliación entre intereses políticos que permita una contribución universal y real a la lucha contra el cambio climático.

\section{La CMNUCC y su operacionalización en el Protocolo de Kioto}

Debido a su carácter marco, la CMNUCC solo estableció objetivos, principios y obligaciones generales, dejando la adopción de obligaciones específicas respecto de la reducción de emisiones de gases de efecto invernadero a las negociaciones posteriores en el marco de las COP. Esencial en estas negociaciones ha sido la discusión en torno a la operacionalización del principio de las responsabilidades comunes pero diferenciadas — conocido como CBDR por sus siglas en inglés common but differentiated responsibilities - , que la CMNUCC retoma de la Declaración de Río.

La CMNUCC consagra el principio de CBDR bajo la lógica de la responsabilidad de todos los países en la lucha contra el cambio climático, que constituye una "preocupación común de toda la humanidad" (preámbulo), pero afirma, a su vez, la contribución distinta que han realizado los países al origen del problema, de lo que se deduce una diferencia en las obligaciones relativas a la respuesta (preámbulo y artículos 3 y 4). En la práctica, esto se tradujo en la adopción dentro del texto de una clasificación rígida entre países desarrollados y países en desarrollo sobre la base de una enumeración nominal en dos anexos. El anexo 1 enumera los países que en aquel momento eran miembros de la Organización para la Cooperación y el Desarrollo Económicos (OCDE) y la entonces Comunidad Europea (en la actualidad Unión Europea), así como países en transición hacia una 
economía de mercado (como Rusia), que deben liderar la acción en materia de mitigación conforme a la CMNUCC. El anexo 2 constituye una subcategoría que contiene solo a los países entonces miembros de la OCDE y la Unión Europea, que asumen principalmente obligaciones en términos de financiamiento. El resto de los países —en la práctica, los países en desarrollo— han sido categorizados como países no pertenecientes al anexo 1 .

La implementación de la CMNUCC mediante metas obligatorias de reducción de emisiones se logró, en primer lugar, con la adopción del Protocolo de Kioto en la COP3 de 1997. Este retomó el sistema de anexos de la CMNUCC, estableciendo obligaciones internacionales de reducción de emisiones solo respecto de los países enumerados en el anexo 1 y eximiendo de toda meta internacional de reducción a los países no enumerados —incluyendo, por ejemplo, a China e India, que se unieron progresivamente a la lista de principales emisores de gases de efecto invernadero a nivel mundial, China reemplazando a Estados Unidos como líder mundial en 2005 (Agencia Internacional de la Energía, s. f.) —. Así, la aplicación del principio de CBDR en el Protocolo de Kioto prolongó y acentuó la diferenciación rígida y estricta iniciada con la CMNUCC en las obligaciones internacionales de los Estados en el régimen jurídico de lucha contra el cambio climático.

A los canales normativos paralelos, intrínsecos al sistema de anexos del Protocolo de Kioto, se sumó la ausencia de ratificación de Estados Unidos, país del anexo 1 que, al momento de adopción del instrumento, lideraba las emisiones a nivel mundial (en la actualidad ocupa el segundo lugar). Esto se debió, en gran medida, al desequilibrio producido por la diferencia normativa en términos de obligaciones de reducción de emisiones entre países desarrollados y países en desarrollo, particularmente con relación a China. Este argumento fue retomado por Canadá, país del anexo 1, cuando anunció en 2011, en el marco de las negociaciones de un segundo ciclo de obligaciones de reducción de emisiones post-Kioto, su retiro del protocolo, aludiendo, entre otros, a la falta de obligaciones tanto de Estados Unidos como de China, líderes en materia de emisiones. 
Así, a pesar de la participación universal que logró la CMNUCC, su operacionalización mediante el Protocolo de Kioto no logró ni un enfoque ni una participación que permitiera abordar el problema del cambio climático desde una perspectiva global. Las negociaciones en torno a un ciclo posterior de reducción de emisiones para prolongar el formato de Kioto solo reflejaron la exacerbación de los desacuerdos en torno al esquema rígido de diferenciación manifestada, por ejemplo, en la oposición de Japón y Rusia a un acuerdo sobre un segundo ciclo de obligaciones. Esta situación fue confirmada por la difícil entrada en vigor de este segundo ciclo, adoptado mediante la Enmienda de Doha al Protocolo de Kioto de 2012. Debido a la dificultad para lograr el número requerido de ratificaciones, la enmienda solo logró entrar en vigor, de manera simbólica, el 31 de diciembre de 2020, es decir, su último día de vigencia. En la práctica, fue reemplazada por el Acuerdo de París, adoptado en 2015.

Ante la difícil situación encontrada en el marco del Protocolo de Kioto y de la Enmienda de Doha, el Acuerdo de París cambia la lógica utilizada hasta ese entonces, intentando establecer un régimen mundial de reducción de emisiones. El acuerdo se hace así cargo de la globalidad del problema, de los cambios en los países que lideran el número de emisiones, de la diversidad creciente de intereses políticos - particularmente visible entre países en desarrollo-y de los avances en la evidencia científica que han acentuado la urgencia de una acción eficaz.

\section{La conciliación en el Acuerdo de París mediante soluciones híbridas}

En base a la experiencia de Kioto y apoyándose en ciertos aspectos en las lógicas introducidas en el Acuerdo de Copenhague y los Acuerdos de Cancún -instrumentos de soft law adoptados en las COP15 y COP16-, el Acuerdo de París, adoptado en la COP21, retiene soluciones híbridas. Este acuerdo reafirma el principio de CBDR en el régimen jurídico de lucha contra el cambio climático, pero flexibiliza su aplicación principalmente mediante dos formas. Por una parte, el texto abandona el 
sistema rígido de anexos basado en la enumeración de países y lo reemplaza por la distinción, más flexible, entre países desarrollados y países en desarrollo, mencionando también algunas subcategorías de países como los países menos desarrollados. A esta flexibilidad se suma la aplicación del principio de CBDR "a la luz de las diferentes circunstancias nacionales" (preámbulo y artículos 2 y 4), lo que introduce una mayor fluidez en la distinción entre ambas categorías de países y se orienta hacia un sistema de autodiferenciación. Por otra parte, si bien se reafirma el principio de CBDR, el Acuerdo de París introduce la obligación de todos los países — desarrollados y en desarrollode establecer metas de reducción de emisiones con miras al objetivo común de mantener el aumento de la temperatura media mundial muy por debajo de los $2{ }^{\circ} \mathrm{C}$ respecto de los niveles preindustriales e idealmente a $1,5^{\circ} \mathrm{C}$. Mediante una operacionalización distinta del principio de CBDR, el acuerdo flexibiliza la lógica de diferenciación rígida entre países de la CMNUCC y del Protocolo de Kioto.

La obligación de carácter universal se manifiesta mediante la preparación, presentación y comunicación de contribuciones determinadas a nivel nacional — NDC, por sus siglas en inglés nationally determined contributions - en materia de reducción de emisiones (artículos 3 y 4). A diferencia del Protocolo de Kioto, las metas no son determinadas a nivel internacional sino a nivel nacional, con miras a alcanzar el objetivo común establecido por el tratado que apuesta al avance colectivo mediante la actualización progresiva, realizada por cada país, de las NDC al alza. A su vez, a diferencia del Protocolo de Kioto, el Acuerdo de París no incluye una obligación de alcanzar las metas definidas a nivel nacional, por lo que las obligaciones respecto de las NDC son esencialmente de medios, no de resultado. Así, en materia de mitigación, el contenido del acuerdo integra una mezcla de herramientas jurídicas diversas, en que la elección es el reflejo de la factibilidad de lograr acuerdos políticos sobre normas aplicables a todos los países.

Dado que el Acuerdo de París no incluye metas obligatorias de reducción de los países, el cambio ha sido descrito como una "desinternacionalización" del régimen jurídico de lucha contra el cambio climático (Dupuy \& Viñuales, 2018). No obstante, si bien es cierto 
que la determinación a nivel nacional de las metas matiza el rol del régimen internacional, el tratado sí establece obligaciones internacionales de carácter más bien procedimental y de medios en materia de mitigación. En este contexto, más que una desinternacionalización, el Acuerdo de París aplica un enfoque híbrido entre una lógica nacional e internacional, en que la arquitectura establecida por el tratado mezcla un sistema bottom-up (de abajo hacía arriba) con elementos top-down — de arriba a bajo- (Falkner, 2016; Bodansky et al., 2017).

Se podría argumentar que la ausencia de obligación internacional respecto de las metas de reducciones y de su cumplimiento constituye un retroceso respecto del marco jurídico establecido por el Protocolo de Kioto basado esencialmente en normas vinculantes. No obstante, el sistema híbrido del Acuerdo de París se hace cargo de la necesidad de conciliar los intereses políticos divergentes para lograr una eficacia real del régimen jurídico, que se ha visto reflejada en una ratificación prácticamente universal del tratado y una entrada en vigor extremadamente rápida, tras menos de un año de adopción. De particular importancia en este sistema híbrido es la inclusión, en la actualidad, de obligaciones de mitigación de los mayores emisores de gases de efecto invernadero, entre los cuales lideran la lista China, Estados Unidos e India. El Acuerdo de París logra así terminar con círculos normativos reducidos, constituyendo un avance fundamental hacia un régimen que permita abordar, en lo concreto, el cambio climático como problema global.

A su vez, si bien el Acuerdo de París no establece el contenido de las metas de reducción de cada país, el tratado constituye un instrumento de derecho duro, jurídicamente vinculante, al igual que el Protocolo de Kioto, diferenciándose de otros instrumentos negociados en los años 2000, como el Acuerdo de Copenhague y los Acuerdos de Cancún. Se debe destacar, sin embargo, que estos últimos instrumentos permitieron lograr avances que fueron fundamentales para la adopción posterior del Acuerdo de París, demostrando la utilidad del soft law en los procesos normativos. Mediante los acuerdos de Copenhague y Cancún, se introdujeron progresivamente los matices al sistema de 
diferenciación, la lógica de abajo hacia arriba en las acciones de mitigación y el objetivo global de limitar el aumento de la temperatura por debajo de los $2^{\circ} \mathrm{C}$. A través de la utilización del soft law en un momento en que un acuerdo internacional jurídicamente vinculante no era factible políticamente, ambos instrumentos contribuyeron a un proceso de creación de derecho duro que desembocó en el Acuerdo de París, demostrando las interacciones entre derecho duro y derecho blandoderecho.

Por su parte, el carácter jurídicamente vinculante del Acuerdo de París ha permitido establecer obligaciones internacionales respecto de las NDC, pero matizar, a su vez, en el contenido de sus disposiciones aquellas que establecen obligaciones para los Estados. Se incorpora así un margen de discreción de los Estados y una flexibilidad en la implementación, que refleja la escala de normatividad ofrecida por el derecho internacional mediante herramientas jurídicas diversas. La introducción de mayor obligatoriedad en el contenido se hubiese visto acompañada de una exigencia de mayor diferenciación por parte de países en desarrollo que constituyen los principales emisores, mientras que un mayor nivel de diferenciación, que hubiese prolongado la lógica del Protocolo de Kioto, hubiese sido difícilmente aceptado por los países desarrollados. El equilibrio logrado en las disposiciones explica que el texto consensuado debe interpretarse en su conjunto, mediante la lógica del paquete todo incluido, en tanto la lectura integrada de las normas refleja la conciliación entre intereses políticos diversos.

Asimismo, el carácter jurídicamente vinculante del tratado en su conjunto otorga resguardos jurídicos ante cambios políticos domésticos, diferenciándose en este sentido del soft law. Este resguardo se refleja emblemáticamente en los requisitos de tiempo para concretar el retiro del Acuerdo de París, los cuales impidieron al gobierno de Donald Trump operacionalizar la denuncia del tratado durante cuatro años y permitieron la reintegración expedita de Estados Unidos bajo el gobierno de Joe Biden.

Así, bajo una forma obligatoria pero con un contenido híbrido que incorpora una "ambigüedad constructiva" (Bodansky \& Rajamani, 
2018), el Acuerdo de París refleja el beneficio de utilizar la variedad de herramientas jurídicas que ofrece el derecho internacional. Las elecciones dependen, en definitiva, del objetivo que se persigue con el instrumento y con cada una de sus disposiciones, así como de la factibilidad de lograr avances en un momento determinado bajo una aceptación global de consenso, en función de los intereses políticos diversos.

\section{Conclusión}

Iniciado en 1992 con la CMNUCC, el régimen jurídico internacional de lucha contra el cambio climático y las negociaciones relativas a su implementación y adaptación progresiva han reflejado uno de los desafíos centrales de la acción internacional actual: lograr acuerdos globales que concilien intereses políticos diversos, en ocasiones profundamente divergentes, sin sacrificar la necesidad de un impacto real. Para esto, distintas técnicas de negociación y una diversidad de instrumentos jurídicos han sido utilizados desde la adopción de la CMNUCC con resultados diversos. Las lecciones de esta experiencia de casi tres décadas se han visto en gran parte reflejadas en el Acuerdo de París que, mediante soluciones híbridas que utilizan la variedad de herramientas que ofrece el derecho internacional, constituye el resultado de equilibrios delicados y factibles entre intereses políticos diversos.

En lo normativo, si bien puede criticarse la ausencia de metas específicas de cada país a nivel internacional, el Acuerdo de París logra avanzar en la necesidad de generar acuerdos globales para hacer frente a un desafío mundial. El instrumento se hace cargo de la conciliación entre la urgencia de elaborar una acción internacional ambiciosa ante el cambio climático y la aceptación global necesaria para que esta ambición se traduzca en un impacto real. En lo concreto, como es el caso habitual en derecho internacional, el balance respecto de la utilidad de las soluciones híbridas introducidas por el Acuerdo de París dependerá de la voluntad de los Estados para avanzar de manera progresiva en la formulación e implementación de las NDC, así como en las negociaciones para adoptar reglas precisas de operacionalización del acuerdo. Así, si bien el derecho internacional 
ofrece herramientas diversas que han sido efectivamente empleadas en las negociaciones del régimen internacional de lucha contra el cambio climático, la eficacia de su utilización dependerá siempre, en último lugar, de la voluntad política para avanzar hacia un impacto real de la acción global.

\section{Referencias}

Acuerdos de Cancún (2010). https://unfccc.int/resource/docs/2010/ cop16/spa/07a01s.pdf

Acuerdo de Copenhague (2009). https:/ /unfccc.int/resource/docs/2009/ cop15/spa/11a01s.pdf

Acuerdo de París (2015). https://treaties.un.org/doc/Treaties/ 2016/02/20160215\%2006-03\%20PM/Ch_XXVII-7-d.pdf

Agencia Internacional de la Energía. (s. f.). Global GHG emissions. https:// www.iea.org/reports/greenhouse-gas-emissions-from-energy-overview/global-ghg-emissions

Bodansky, D., \& Rajamani, L. (2018). The issues that never die. Carbon and Climate Law Review, 12(3), 184-190. https://cclr.lexxion.eu/article/ CCLR/2018/3/4

Bodansky, D., Brunnée, J., \& Rajamani, L. (2017). International Climate Change Law. Oxford University Press.

Boyle, A., \& Ghaleigh, N. S. (2016). Climate change and international law beyond the UNFCCC. En C. P. Carlarne, K. R. Gray, \& R. Tarasofsky (Eds.), The Oxford Handbook of International Climate Change Law (pp. 26-53). Oxford University Press.

Carbon Brief. (s. f.). Interactive: the UNFCCC negotiating alliances. https:// www.carbonbrief.org/interactive-the-negotiating-alliances-at-theparis-climate-conference

Convención Marco de las Naciones Unidas sobre el Cambio Climático (1992). https://treaties.un.org/doc/Treaties/1994/03/19940321\%20 04-56\%20AM/Ch_XXVII_07p.pdf

Declaración de Río sobre el Medio Ambiente y Desarrollo (1992). https:// www.un.org/spanish/esa/sustdev/agenda21/riodeclaration.htm

Dupuy, P., \& Viñuales, J. (2018). International Environmental Law. Cambridge University Press. 
Falkner, R. (2016). The Paris Agreement and the new logic of international climate politics. International Affairs, 92(5), 1107-1125. https:// academic.oup.com/ia/article-abstract/92/5/1107/2688148?redirectedFrom $=$ fulltext

Pellet, A. (2018). Les raisons du développement du soft law en droit international: choix ou nécessité? En P. Deumier \& J.-M. Sorel (Eds.), Regards croisés sur la soft law en droit interne, européen et international (pp. 177-192). LGDJ/Lextenso. https://4e8d1502-3afc-456b-9cc2-86f62537d292. filesusr.com/ugd/90698f_93005d9df6394dd29be9a378198ac57a.pdf

Protocolo de Kioto de la Convención Marco de las Naciones Unidas sobre el Cambio Climático (1997). https://treaties.un.org/doc/Treaties/1998/09/19980921\%2004-41\%20PM/Ch_XXVII_07_ap.pdf

Rajamani, L. (2016). The 2015 Paris Agreement: Interplay between hard, soft, and non-obligations. Journal of Environmental Law, 28(2), 337-358.

Rajamani, L. (2020), Innovation and experimentation in the international climate change regime. Brill/Nijhoff. 